of the observed portion of the inner corona, the bolometric effect of its visual radiation may be supposed to be equal to that of the latter; but the observations above recorded show that the total radiations from the moon being $55+30$, or eighty-five bolometric divisions, are seventeen times as great as the radiations from the inner corona, and hence it may be supposed that the corora lacks that large amount of infra-red radiation which is proper to the moon's spectrum.

The moon's spectrum, however, is that of a heated solid body, and all heated solid bodies, and heated gaseous bodies as well, send to the bolometer large amounts of infra-red radiation. So far, then, we might conclude that the inner corona has not the radiations of a hot solid or gaseous body, but, owing to the lack of a contemporary measure of the sky radiation just outside the corona, and of a full knowledge of the influences that the atmospheric radiations would have on our ability to discriminate this, the above conclusions seemed to me only probable, and worth verification at the forthcoming eclipse.

Smithsonian Institution, April 29.

\author{
S. P. LANGley.
}

\section{The Persistence of the Spectrum of Carbon Monoxide.}

THE letter of Dr. Carl v. Wesendonk (p. 29), which gives an account of the spectrum of carbon monoxide appearing in a vacuum tube containing silicon tetrafluoride, affords an instance of the extreme difficulty of obtaining vacuum tubes charged with perfectly pure substances. The case he cites of silicon fluoride being prepared from "pure" sulphuric acid, glass and fluor spar, without any but glass joints to connect the different parts of the apparatus, is one in which neither the perfect freedom of the sulphuric acid, nor of the glass itself, from carbon compounds can be relied upon. In experiments on the absorption spectrum of ozone made by me in $188 \mathrm{I}$, it was found that strong sulphuric acid free from all the usual impurities was not absolutely clear, but by being kept in an atmosphere containing a large proportion of ozone it became perfectly brilliant and absolutely colourless when seen in volumes of half a gallon to two gallons at a time. It appeared from further experiments that the impurities were either carbon or some form of organic matter probably coming from dust or dirt. As to the purity of the glass used for vacuum tubes, it may be remarked that dust and condensed vapour from carbonaceous matter, such as the products of combustion from lamp oil or coal, adheres to its surface with much tenacity. It is probable that the fluor spar contained organic matter, for the reason that this substance is associated with limestone of a bituminous character in England and that it has been asserted that its colour is due to organic substances. By the action of sulphuric acid a gaseous carbon compound might easily be evolved which would contaminate the silicon fluoride even if there were no carbonates present. Next we have to consider the traces of air which may remain in the tube, and must not regard these as being absolutely free from hydrocarbons. M. Armand Gautier has shown that there are combustible gases in the atmosphere, one of which is a hydrocarbon, the other hydrogen, and there is also some carbon monoxide. The difficulty of removing these by ordinary chemical treatment is so great that special operations and reagents were provided for their removal.

In vacuum tubes it is known that carbon monoxide shows its spectrum brilliantly when the pressure is extremely low, and that subsequently it disappears. The very interesting research of Prof. Smithells on "The Spectra of Carbon Compounds," in the April number of the Phil. Mag., illustrates this. Furthermore, it shows distinctly that the same spectrum is obtainable from both carbon monoxide and carbon dioxide (loc. cit. pp. 489 and 490). We know, too, from the experiments of Regnault and of Bunsen on the analysis of atmospheric air, that carbon dioxide is absorbed by glass. In view of the facts quoted by Prof. Smithells, the carbon monoxide spectrum is, in his opinion, really due to carbon dioxide, but this latter may easily be decomposed into carbon monoxide and oxygen under the influence of the spark discharge.

The Swan spectrum, attributed variously to a hydrocarbon and to the element carbon by previous investigators, is, according to Smithells, to be attributed to carbon monoxide. It appears also in Dr. v. Wesendonk's letter that when the glass tubes in which the electrodes were fused had become heated, the carbon monoxide spectrum was faintly visible. This would be quite in accordance with the probability that carbon dioxide was evolved from the glass.

$$
\text { NO. I } 646 \text {, VOL. } 64]
$$

A tube containing silicon bydride also showed the carbon monoxide and the Swan spectrum, as well as hydrogen and mercury lines, but no silicon lines were observable. Considering all the facts of the case, it is not conceivable that the spectra in question arise in any way from the decomposition or dissociation of the silicon in the compound, either in the state of vapour as fluoride, of gas as hydride, or in the solid state as glass.

April 25.

W. N. Hartley.

\section{The Use of "Axis-vectors."}

THE effort to popularise the elements of vector algebra is commendable. The power and the direct insight conferred by the use of vector quantities should be sought consistently in the study of physics; and it is true that the introduction of these methods has been needlessly postponed. But it lies in the very nature of such benefits that they are not to be secured except upon tenable grounds and as the result of a continuous argument. If a particular quantity is to be classed with vectors, that cannot be done upon a basis which is reducible to the bare statement: "This magnitude may be represented by a straight line of given direction and length ; therefore it is a vector." Witness, for example, moment of inertia, which is not properly a vector, although its magnitude can be associated with a rotation-axis Vector quantities must be subject to the process of "geometrical addition"; there is a total obtainable as the vector sum of con. stituent parts. This is equivalent to saying that there is a greatest value $Q$ (resultant) for one direction, and that the law of orthogonal projection applies. Thus the value $Q_{\mathrm{I}}$ for any other direction must satisfy the equation

$$
Q_{1}=Q \cos \left(Q_{1}, Q\right) \text {. }
$$

This projective property must be proved somehow in each case.

The conception of a vector is usually established as an elementary matter with the aid of instances like velocity and force. Velocity is so closely connected with linear displacement that the operations of geometrical addition and projection can be almost intuitively recognised as valid for both quantities. The graphical representation of forces, and the application to them of the " parallelogram construction," can be approached from the experimental side, furnishing a timely reminder that this procedure (as regards physical quantity) is ultimately justified by appeal to phenomena. The inclusion of "axis-vectors" $(e . g$. angular velocity and acceleration; moments of force and of momentum) in the class is a second step, of no less importance than the first. The proofs put forward to cover this extension of the thought afford fruitful material to the student of applied logic, through their variations of scope and emphasis. The analysis of some demonstrations now current prompts the remarks which follow.

First, linear vectors, like velocity, force, magnetic field, have what may be termed objective direction. But direction is assignable to axis-vectors by usage only, in the line of a (possible or actual) rotation-axis. Further, the sense in this line is arbitrary, being determined, for example, by the " rule of the righthanded screw." This double convention underlying the graphical representation of axis-vectors must be insisted upon.

Secondly, the theorem known as the "parallelogram of angular velocities" is really intended to prove that the linear velocities of all points in a rigid body satisfy the conditions of rotation in certain cases. The characteristic of rotation is a relation to the axis as regards the direction and the magnitude of all velocities, usually expressed as $v=r \omega, v$ being perpendicular to both $r$ and the rotation-axis. The proof of the theorem is only implicitly complete, if we content ourselves with showing that simultaneous angular velocities about intersecting axes produce zero linear velocity on a particular line. And the corollary covering the most important point is often not even mentioned. Similar considerations apply to angular acceleration.

Thirdly, the direct graphical representation of force-moment is connected with areas and not with lines. These areas are in general parallelograms, with adjacent sides representing the force and the distance of its point of application from a chosen point on the rotation-axis. The fundamental case is that in which the parallelogram is perpendicular to the axis, and its area shows the moment for a line through one vertex. For an oblique axis through the same vertex, the moment is obtained by projecting that area upon a plane perpendicular to the new axis. This follows easily from the definition of force-moment. 